\title{
Depairing currents in the superconductor/ferromagnet proximity system $\mathrm{Nb} / \mathrm{Fe}$
}

\author{
J. M. E. Geers, M. B. S. Hesselberth, and J. Aarts* \\ Kamerlingh Onnes Laboratory, Leiden Institute of Physics, Leiden University, P.O. Box 9504, NL-2300 RA Leiden, The Netherlands
}

\author{
A. A. Golubov
}

University of Twente, P.O. Box 217, NL-7500 AE Enschede, The Netherlands

(Received 10 January 2001; published 7 August 2001)

\begin{abstract}
We have investigated the behavior of the depairing current $J_{d p}$ in ferromagnet/superconductor/ferromagnet $(F / S / F)$ trilayers as function of the thickness $d_{s}$ of the superconducting layers. Theoretically, $J_{d p}$ depends on the superconducting order parameter or the pair-density function, which is not homogeneous across the film due to the proximity effect. We use a proximity-effect model with two parameters (proximity strength and interface transparency), which can also describe the dependence of the superconducting transition temperature $T_{c}$ on $d_{s}$. We compare the computations with the experimentally determined zero-field critical current $J_{c 0}$ of small strips (typically $5-\mu \mathrm{m}$ wide) of $\mathrm{Fe} / \mathrm{Nb} / \mathrm{Fe}$ trilayers with varying thickness $d_{\mathrm{Nb}}$ of the $\mathrm{Nb}$ layer. Near $T_{c}$ the temperature dependence $J_{c 0}(T)$ is in good agreement with the expected behavior, which allows extrapolation to $T=0$. Both the absolute values of $J_{c 0}(0)$ and the dependence on $d_{\mathrm{Nb}}$ agree with the expectations for the depairing current. We conclude that $J_{d p}$ is correctly determined, notwithstanding the fact that the strip width is larger than both the superconducting penetration depth and the superconducting coherence length, and that $J_{d p}\left(d_{s}\right)$ is correctly described by the model.
\end{abstract}

DOI: 10.1103/PhysRevB.64.094506

PACS number(s): 74.50.+r, 74.76.-w, 74.80.- $-\mathrm{g}$

\section{INTRODUCTION}

A still relatively little explored area of research in nonequilibrium superconductivity concerns phenomena involving spin-polarized quasiparticles. Pioneering work on spinpolarized tunneling in conventional $s$-wave superconductors was performed by Meservey and Tedrow, ${ }^{1}$ who studied different ferromagnets $(F)$ in $F / \mathrm{Al}_{2} \mathrm{O}_{3} / \mathrm{Al}$ tunnel junctions and found that the tunnel current can show varying degrees of spin polarization. More recently, experiments were performed by different groups in order to establish whether superconductivity can be suppressed by injecting spinpolarized quasiparticles. ${ }^{2-4}$ In these cases the combinations existed of a $d$-wave high- $T_{c}$ superconductor $\left(\mathrm{XBa}_{2} \mathrm{Cu}_{3} \mathrm{O}_{7}\right.$, with $X=\mathrm{Y}$, Dy) and a fully spin-polarized ferromagnetic manganite $\left(A_{0.67} B_{0.33} \mathrm{MnO}_{3}\right.$ with $A=\mathrm{La}, \mathrm{Nd}$ and $\left.B=\mathrm{Ca}, \mathrm{Sr}\right)$, either with or without a barrier of a different oxide; measured was the change in the zero-field critical current density $J_{c 0}$ of the superconducting films upon applying a current bias through the ferromagnet. The results are not fully conclusive, and certainly not quantitative. Although generally a suppression of $J_{c 0}$ was observed, heating effects could not always be fully ruled out since the manganites are highly resistive metals (see the discussion in Ref. 4), and the geometry did not always allow to determine the area of the current injection, and therefore the injected current density. Moreover, since $J_{c 0}$ in high- $T_{c}$ superconductors generally is not the depairing current $J_{d p}$ but involves flux motion, $J_{c 0}$ is not a direct measure for the amount of depression of the superconducting order parameter. To our knowledge, similar experiments have not been performed with combinations of conventional metals, although that would have some clear advantages. The interpretation of results would not be complicated by, e.g., inhomogeneous currents in the ferromagnet or anisotropic gaps in the superconductor; lithographic techniques could be brought to bear in order to have well-defined superconducting bridges and injection contacts; and it should be possible to identify the effects of the spin-polarized quasiparticles on $J_{d p}$.

Still, two points deserve special interest. The first is that, in planning such an $F / I / S$ experiment, there is the potential problem of insufficient knowledge of the tunneling process. This was already apparent in the work of Meservey and Tedrow cited above ${ }^{1}$ since the experiments always showed a positive sign for the spin polarization, even in the cases of, e.g., Co and $\mathrm{Ni}$ where a negative sign was expected. Recently, this was explained by demonstrating that the choice of barrier material can strongly influence and even reverse the spin polarization of the tunneling current, ${ }^{5}$ with obvious consequences for the interpretation of the injection experiments. It may be advantageous to also contemplate an $(F$ or $N) / I / F / S$ configuration; in this case the barrier is only used to increase the energy of the electrons coming from an $N$ or $F$ contact, while the polarization now takes place in a thin $F$ layer between barrier and superconductor. The disadvantage here is that the $F$ layer in connection with the superconductor will suppress the order parameter and therefore $J_{d p}$ in the $S$ layer. Still, since the proximity effect for $S / F$ systems is understood reasonably well, at least with respect to the behavior of the order parameter in the $S$ layer, ${ }^{6}$ the effect on $J_{d p}$ may also be quantifiable. The second point for consideration is that even in the case of conventional superconductors the determination of $J_{d p}$ need not be straightforward. The difficulty lies in the fact that the superconducting bridge must have a width of no more than both the superconducting penetration depth $\lambda$ and the superconducting coherence length $\xi$. The first is needed to avoid current pile up near the edges (as a consequence of screening of the self field), the second is required in order to avoid vortex nucleation and flow, which gives rise to dissipation before $J_{d p}$ is reached. 
These conditions can be met, e.g., for $\mathrm{Al}$, which has a Bardeen-Cooper-Schrieffer coherence length $\xi_{0}$ of about $1.5 \mu \mathrm{m}$, while $\lambda$ can also be made of the order of $1 \mu \mathrm{m}$ by making the film thin enough. For Al-bridges of less than $1-\mu \mathrm{m}$ wide it was shown by Romijn et al. ${ }^{7}$ that the measured $J_{d p}$ agreed very well with the theoretical calculations by Kupriyanov and Lukichev ${ }^{8}$ based on the Eilenberger equations and therefore valid in the whole temperature regime below $T_{c}$. For a material such as $\mathrm{Nb}$, with $\xi_{0}$ and $\lambda$ of the order of $50 \mathrm{~nm}$, such agreement need not be expected.

In this paper we show that, at least close to $T_{c}$, the values of the zero-field critical current $J_{c 0}$ measured on bridgestructured $\mathrm{Nb}$ samples are essentially the values expected for the depairing current. Furthermore, we measure the depression of $J_{c 0}$ in trilayers of $\mathrm{Fe} / \mathrm{Nb} / \mathrm{Fe}$, as a function of the thickness $d_{\mathrm{Nb}}$ of the $\mathrm{Nb}$ layer. We compare the behavior of $J_{c 0}\left(d_{\mathrm{Nb}}\right)$ with the behavior of $T_{c}\left(d_{\mathrm{Nb}}\right)$, and also with calculations of the proximity effect and the pair-breaking velocity using a two-parameter formalism based on the Usadel equations. We find that $J_{c 0}\left(d_{\mathrm{Nb}}\right)$ is well described by the same two parameters that describe the behavior of $T_{c}\left(d_{\mathrm{Nb}}\right)$. The conclusion is that the suppression of the depairing current as a consequence of the depression of the order parameter in $S / F$ structures can be well described by proximity-effect theory, making $(F, N) / I / F / S$ injection experiments a distinct possibility.

\section{DEPAIRING CURRENT: THEORY}

Close to $T_{c}$, the classical Ginzburg-Landau (GL) result for the temperature dependence of the depairing current of a thin film, under the assumption of a homogeneous superconducting order parameter over the film thickness, is given by

$$
J_{d p}^{G L}(t)=J_{d p}^{G L}(0)(1-t)^{3 / 2},
$$

with $t=T / T_{c}$. The prefactor $J_{d p}$ is of the order of $H_{c} / \lambda$, with $H_{c}$ the thermodynamic critical field, and will be given more precisely below. For arbitrary temperatures, calculations were performed by Kupriyanov and Lukichev, who essentially solved the Eilenberger equations for a superconductor carrying a current, with the velocity of the condensate leading to a phase gradient. ${ }^{8}$ Their results recover the GL behavior near $T_{c}$ :

$$
J_{d p}^{G L}(t)=\frac{16}{9 \sqrt{7 \zeta(3)}} \sqrt{\chi\left(\rho_{d}\right)}\left[e N(0) v_{F} k_{B} T_{c}\right](1-t)^{3 / 2} .
$$

Here, the constants have their usual meaning, $N(0)$ is the density of states at the Fermi level per spin direction, and $\chi\left(\rho_{d}\right)$ is the G'orkov function controlled by the "dirt parameter" $\rho_{d}=\left(\hbar v_{F}\right) /\left(2 \pi k_{B} T_{c} l_{e}\right)$, with $l_{e}$ the electronic mean free path. In the dirty limit, $\left(\rho_{d} \rightarrow \infty\right) \chi\left(\rho_{d}\right) \rightarrow 1.33 l_{e} / \xi_{0}$, this becomes

$$
J_{d p}^{G L}(0)=1.26\left[e N(0) v_{F} \Delta(0)\right] \sqrt{\frac{l_{e}}{\xi_{0}}},
$$

which is equivalent to the expression given by Romijn et al. ${ }^{7}$

$$
J_{d p}^{G L}(0)=\frac{16 \pi^{2} \sqrt{2 \pi}}{63 \zeta(3)}\left[e N(0) v_{F} k_{B} T_{c}\right] \sqrt{\frac{k_{B} T_{c} l_{e}}{\hbar v_{F}}},
$$

which can also be written in terms of experimental parameters as

$$
J_{d p}^{G L}(0)=7.84\left[\frac{\left(k_{B} T_{c}\right)^{3}}{e^{2} \hbar v_{F}(\rho l)} \frac{1}{\rho}\right]^{1 / 2} .
$$

This way of writing also emphasizes the proportionality $J_{d p}^{G L}(0) \propto \sqrt{1 / \rho}$, since the product $\rho l$ is a materials constant. At low temperatures the value of $J_{d p}$ saturates, reaching a zero-temperature value of

$$
\begin{aligned}
J_{d p}(0) & =1.491 e N(0) \sqrt{\frac{D}{\hbar}} \Delta^{3 / 2}(0) \\
& =0.486\left[\operatorname{eeN}(0) v_{F} \Delta(0)\right] \sqrt{\frac{l_{e}}{\xi_{0}}},
\end{aligned}
$$

with $D=1 / 3 v_{F} l_{e}$ the diffusion constant. Comparison with Eq. (3) shows that the ratio between the saturation value and the GL-extrapolated value equals $J_{d p}(0) / J_{d p}^{G L}(0)=0.385$. In the case of $F / S$ (or $N / S$ ) multilayers, the superconducting order parameter is depressed near the interfaces, and this has to be taken into account in calculating $J_{d p}$. For this we use the proximity-effect model, based on the Usadel equations (dirty limit conditions), that was also used for calculating the depression of $T_{c}$ with decreasing thickness of the superconductor. ${ }^{6}$ Details will be given in the Appendix but here we briefly introduce the main parameters of the theory. In principle, the shape of the order parameter on both sides of the interface depends on the bulk transition temperature $T_{c 0}$, on the coherence lengths $\xi_{S, F}$, on the normal-state resistivities $\rho_{S, F}$, and on the transparency $T$ of the interface. From the boundary conditions for the order parameter [see Eqs. (A4)] it follows that, apart from $T_{c 0}$, only two independent parameters are needed, the proximity strength parameter $\gamma$ and the transparency parameter $\gamma_{b}$. The value of $\gamma$ $=\left(\xi_{S} \rho_{S}\right) /\left(\xi_{F} \rho_{F}\right)$ can be fully determined from the experiment; the only free parameter is $\gamma_{b}\left(0 \leqslant \gamma_{b} \leqslant \infty\right)$, which is approximately connected to the transparency $T$ (with $0 \leqslant T$ $\leqslant 1)$ by

$$
T=\frac{1}{1+\gamma_{b}} .
$$

As was shown in Ref. 6, in $F / S$ systems, $T$ can be quite low for a high magnetic moment in the $F$ layer, which is presumably due to the suppression of Andreev reflections by the exchange splitting of the spin subbands. Figure 1 gives the results of some typical calculations, performed for the system $\mathrm{Fe} / \mathrm{Nb} / \mathrm{Fe}$ with the appropriate proximity-effect parameters $\gamma=34.6$ and $\gamma_{b}=42$ (see Sec. IV). Shown is $J_{d p}(t)$ for two different thicknesses $\left(d_{S} / \xi_{S}=20,7.5\right)$, normalized on the bulk value $J_{d p}^{b u l k}(0)$ as given by Eq. (6). Note that this involves a factor $\left(T_{c} / T_{c}^{b u l k}\right)^{3 / 2}$. The thickness dependence of $T_{c}$ and the normalized depairing current at $T=0$ (see the inset of Fig. 1) are quite different, with a much stronger 


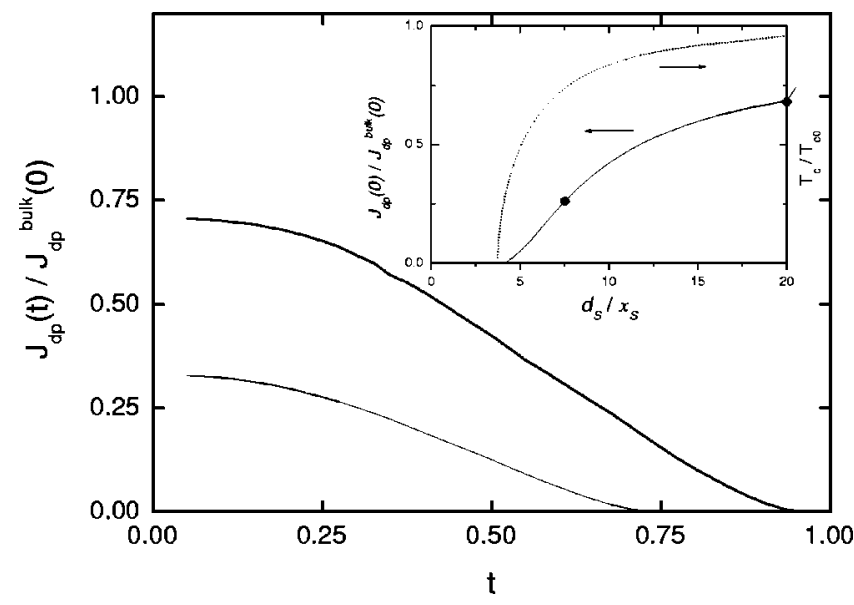

FIG. 1. The temperature dependence of the normalized depairing current $J_{d p}(t) / J_{d p}^{\text {bulk }}(0)$ of an $F / S / F$ trilayer for $S$-layer thicknesses $d_{S} / \xi_{S}=20$ (upper), 7.5 (lower). Parameters typical for Fe/ $\mathrm{Nb} / \mathrm{Fe}$ were used, namely, $\gamma=34.6$ and $\gamma_{b}=42$. Inset: thickness dependences of the normalized depairing current at $T=0$, and of $T_{c}$ of the same trilayer. The black dots are at $d_{S} / \xi_{S}=20,7.5$.

depression of the depairing current at relatively high thickness of the superconductor. This can be qualitatively understood by noting that $T_{c}$ is a measure for the maximum value of the superconducting order parameter in the layer, while the depairing current comes from an average over the layer thickness, which also involves lower values of the order parameter.

\section{EXPERIMENT}

Samples were grown on $\mathrm{Si}(100)$ substrates, by dc sputtering in a system with a base pressure of $10^{-9}$ mbar in an $\mathrm{Ar}$ pressure of $6 \times 10^{-3}$ mbar. Sputtering rates were of the order $0.1 \mathrm{~nm} / \mathrm{s}$ for $\mathrm{Nb}$ and $0.03 \mathrm{~nm} / \mathrm{s}$ for Fe. One series of samples consisted of trilayers $\mathrm{Nb} / \mathrm{Fe} / \mathrm{Nb}$ with $\mathrm{Nb}$ thickness $d_{\mathrm{Nb}}=5 \mathrm{~nm}$ and the Fe thickness $d_{\mathrm{Fe}}$ varying between $2 \mathrm{~nm}$ and $25 \mathrm{~nm}$. These were used to determine the magnetization $M_{\mathrm{Fe}}$ of the $\mathrm{Fe}$ layers in the presence of $\mathrm{Fe} / \mathrm{Nb}$ interfaces with a commercial superconducting quantum interference device based magnetometer. The behavior of $M_{\mathrm{Fe}}$ vs $d_{\mathrm{Fe}}$ could be well described with a straight line, yielding a magnetic moment per Fe atom of $2.36 \mu_{B}$ ( $\mu_{B}$ being the Bohr magneton), slightly above the bulk value of $2.2 \mu_{B}$ and a magnetically dead layer per interface $d_{M D}$ of $0.1 \mathrm{~nm}$. This value is somewhat lower than reported for molecular-beam-epitaxy (MBE)-grown samples ${ }^{9,10}$ and might suggest small interface roughness. However, in an unrelated study of the magnetism and interface roughness of $\mathrm{Nb} / \mathrm{Fe}_{0.77} \mathrm{~V}_{0.23}$ multilayers prepared in the same sputtering system, x-ray diffraction showed a mean roughness of about $0.9 \mathrm{~nm}$ for both the $\mathrm{Nb}$ and the $(\mathrm{Fe}, \mathrm{V})$ layers, with $d_{M D}$ about $0.4 \mathrm{~nm}$. The roughness is quite comparable to what was reported for the MBEgrown $\mathrm{Nb} / \mathrm{Fe}$ samples (around 0.6-0.7 nm). Apparently, the sputtering process leads to similar interface roughnesses as previously reported, which can be expected from the relatively large lattice-parameter mismatch, but possibly to somewhat less interlayer mixing, resulting in a slightly smaller $d_{M D}$. For the critical current experiments, two other series of samples consisted of trilayers of $\mathrm{Fe} / \mathrm{Nb} / \mathrm{Fe}$ with $d_{\mathrm{Fe}}=5 \mathrm{~nm}$ and varying $d_{\mathrm{Nb}}$. One set was structured by Arion etching into strips with a width $w=100 \mu \mathrm{m}$, the other into strips with a width $w=6 \mu \mathrm{m}$, or sometimes $10 \mu \mathrm{m}$ or $20 \mu \mathrm{m}$. In both cases the length between the voltage contacts was $1 \mathrm{~mm}$. The first set (deposited in two different runs) was used for measuring $T_{c}\left(d_{\mathrm{Nb}}\right)$, the second set for both $T_{c}\left(d_{\mathrm{Nb}}\right)$ and $J_{c}\left(d_{\mathrm{Nb}}\right)$. In all cases, the typical width of the resistive transitions to the superconducting state was $50 \mathrm{mK}$.

Also measured were single films of $\mathrm{Fe}$ and $\mathrm{Nb}$ with different strip widths in order to establish values for the specific resistivity $\rho_{\mathrm{Fe}, \mathrm{Nb}}\left(\right.$ at $10 \mathrm{~K}$ ), for $T_{c}$ and for the upper critical field $B_{c 2}(T)$. On average, we find $\rho_{\mathrm{Fe}} \approx 7.5 \mu \Omega \mathrm{cm}, \rho_{\mathrm{Nb}}$ $\approx 3.7 \mu \Omega \mathrm{cm}, T_{c}=9 \mathrm{~K}$, and $S=-d B_{c 2} / d T=0.24 \mathrm{~T} / \mathrm{K}$, yielding $\xi_{G L}(0)=\sqrt{\Phi_{0} /\left(2 \pi S T_{c}\right)}=12.2 \mathrm{~nm}$. This corresponds to $\xi_{S}=7.8 \mathrm{~nm}$. No special precautions were taken to shield residual magnetic fields. The zero-field critical current $I_{c}$ was determined at different temperatures $T$ by measuring current $(I)$-voltage $(V)$ characteristics. For this, a dc current was switched on for the time of the order of $1 \mathrm{~s}$ and the voltage recorded, to prevent heating via the contacts. All samples showed a clear transition from the superconducting to the normal state, with a large and almost instantaneous increase in voltage at $I_{c}$. Upon detecting this rise, the current was also turned off since the sample then started to heat immediately. Most samples also showed a small rise in voltage prior to the major transition, probably due to vortex motion. We shall come back to this point in the discussion. In some instances, we checked whether the values measured for $I_{c}$ depend on the domain state of the $F$ layers by magnetizing them with a large magnetic field (order of $1 \mathrm{~T}$ ). This turned out not to be the case. Important for the theoretically expected value of $J_{d p}(0)$ is the value of the resistivity of the superconducting layer [see Eq. (4)]. This value, $\rho_{\mathrm{Nb}}$, was extracted from the normal state resistance $R_{n}$ at $10 \mathrm{~K}$ of the patterned samples by assuming that the $\mathrm{Nb}$ layer and the 10 -nm-thick Fe layer $\left(\rho_{\mathrm{Fe}}=7.5 \mu \Omega \mathrm{cm}\right)$ contribute as parallel resistors.

The resulting values for $\rho_{\mathrm{Nb}}$ are given in Table I, together with the strip width $w$ and $T_{c}$. The values for the thinner films (around $50 \mathrm{~nm}$ ) are somewhat larger than what we usually find for single $\mathrm{Nb}$ films, and approach that value for the thick films.

\section{RESULTS}

Figure 2 shows the measured values for $T_{c}\left(d_{\mathrm{Nb}}\right)$ for both sample sets, with the the two types of open symbols denoting the two deposition runs for that set, and the solid symbols denoting the samples used for measuring $J_{c 0}$. The overall data spread is small, and the data can be well described by the proximity-effect theory for $S / F$ systems we used for analyzing the behavior of $\mathrm{V} /\left(\mathrm{Fe}_{x} \mathrm{~V}_{1-x}\right)$ in Ref. 6, with the two parameters $\gamma$ and $\gamma_{b}$ defined above. We use the same value for $\xi_{F}$ as in the case of $\mathrm{V} / \mathrm{Fe}, \xi_{\mathrm{Fe}}=0.14 \mathrm{~nm}$ and values for $\xi_{s}, \rho_{F}$, and $\rho_{S}$ as given in Sec. III, yielding $\gamma=34.6$. The best description for $T_{c}\left(d_{\mathrm{Nb}}\right)$ then is for $\gamma_{b}=42$, as shown by the drawn line in Fig. 2. The critical thickness for the $S$ layer 
TABLE I. Parameters of the $\mathrm{Fe} / \mathrm{Nb} / \mathrm{Fe}$ samples and the single $\mathrm{Nb}$ film used for the critical current measurements. Given are the thickness of the Nb layer $d_{\mathrm{Nb}}$, the strip width $w$, the critical temperature $T_{c}$, the calculated specific resistance of the $\mathrm{Nb}$ layer $\rho_{\mathrm{Nb}}$, and the Ginzburg-Landau extrapolated critical current at zero temperature $\quad J_{c 0}^{G L}(0) . \quad S(\mathrm{Nb})=-\mu_{0} d H_{c 2} / d T=0.24 \mathrm{~T} / \mathrm{K}, \quad \xi_{\mathrm{Fe}}$ $=0.14 \mathrm{~nm}, \rho_{\mathrm{Fe}}=7.52 \mu \Omega \mathrm{cm}, \gamma \approx 34.6$, and $\gamma_{b}=42$.

\begin{tabular}{lccccc}
\hline \hline Type & $\begin{array}{c}d_{\mathrm{Nb}} \\
(\mathrm{nm})\end{array}$ & $\begin{array}{c}w \\
(\mu \mathrm{m})\end{array}$ & $\begin{array}{c}T_{c} \\
(\mathrm{~K})\end{array}$ & $\begin{array}{c}\rho_{\mathrm{Nb}} \\
(\mu \Omega \mathrm{cm})\end{array}$ & $\begin{array}{c}J_{c 0}^{G L}(0) \\
\left(10^{11} \mathrm{~A} / \mathrm{m}^{2}\right)\end{array}$ \\
\hline$F / S / F$ & 36 & 6 & 3.63 & 5.97 & 0.522 \\
$F / S / F$ & 40 & 6 & 4.36 & 6.51 & 1.55 \\
$F / S / F$ & 42 & 10 & 5.07 & 10.4 & 1.58 \\
$F / S / F$ & 53 & 10 & 5.62 & 8.08 & 2.64 \\
$F / S / F$ & 60 & 6 & 6.63 & 5.03 & 3.46 \\
$F / S / F$ & 75 & 6 & 7.34 & 4.95 & 6.14 \\
$F / S / F$ & 100 & 6 & 8.05 & 4.58 & 6.86 \\
$F / S / F$ & 150 & 6 & 8.61 & 3.94 & 11.2 \\
$\mathrm{Nb}$ & 53 & 20 & 9.00 & 7.24 & 15.1 \\
\hline \hline
\end{tabular}

for onset of superconductivity $d_{c r}^{S}$ can be taken either from the lowest measured value for $T_{c}$ or from the extrapolated value of the calculated curve, $d_{c r}^{S}=29 \mathrm{~nm}$, corresponding to a ratio $d_{c r}^{S} / \xi_{S}=3.7$, which is somewhat higher than in the case of $\mathrm{V} / \mathrm{Fe}$ where we found 3.2. Apparently, the effect of ferromagnet on superconductor is slightly stronger in the $\mathrm{Nb} / \mathrm{Fe}$ case, but this is not the issue of the current paper. Also note that critical thicknesses of a few times $\xi_{s}$ preclude the possibility of coupling effects between the two magnetic layers. For instance, different directions of the magnetization in the two $F$ layers might give rise to anomalous suppression of superconductivity ${ }^{11,12}$ for a small window of values of both $d_{S} / \xi_{S}$ and $d_{F} / \xi_{F}$ around 1 ; neither condition is fulfilled in our case.

In Fig. $3 J_{c 0}=I_{c 0} /(w d)$ is plotted vs reduced temperature $t=T / T_{c}$ for $d_{\mathrm{Nb}}=42,60$, and $75 \mathrm{~nm}$. All curves show a clear upturn with decreasing temperature in the region close to $T_{c}$, above $t \approx 0.9$. Plotting $J_{c 0}^{2 / 3}(t)$ vs $t$ results in a straight line in

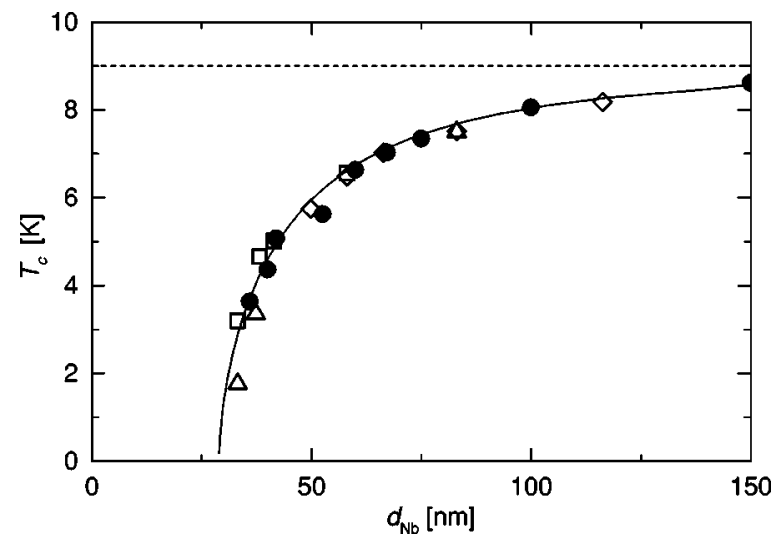

FIG. 2. $T_{c}$ of the different sets of $\mathrm{Fe} / \mathrm{Nb} / \mathrm{Fe}$ trilayers. The solid symbols denote the samples used for the critical current measurements. The line shows the theoretical dependence $T_{c}\left(d_{s}\right)$ for the parameter values $\gamma=34.6$ and $\gamma_{b}=42$.

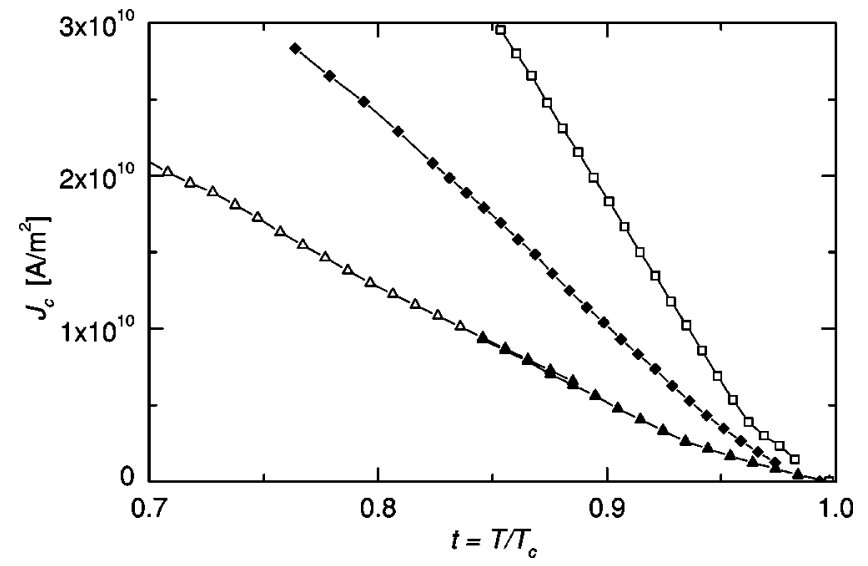

FIG. 3. Experimentally determined critical current density $J_{c 0}$ vs reduced temperature $t=T / T_{c}$ for the $\mathrm{Fe} / \mathrm{Nb} / \mathrm{Fe}$ trilayers with $d_{\mathrm{Nb}}=42 \mathrm{~nm}$ (triangles), $60 \mathrm{~nm}$ (solid diamonds), and $75 \mathrm{~nm}$ (open squares). The solid and open symbols for $d_{\mathrm{Nb}}=42 \mathrm{~nm}$ correspond to measurements with nonpumped and pumped He bath, respectively.

this temperature regime, which can be extrapolated to $t=0$. The ensuing values for $J_{c 0}^{G L}(0)$ are given in Table I for all samples, and comprise some of the main experimental results. They can also be used to normalize the data. Figure 4 shows $\left[J_{c 0}(t) / J_{c 0}^{G L}(0)\right]^{2 / 3}$ vs $t$ together with the line $1-t$ (the GL behavior) and the result of the full theoretical calculation, which is now independent of the parameters. All data collapse on the universal curve above $t=0.9$. At lower temperatures, the thinnest films $\left(d_{s}=36,40,42,53 \mathrm{~nm}\right)$ follow the full calculation quite closely, even down to $t \approx 0.6$. The difference between the data of $36 \mathrm{~nm}$ and $40 \mathrm{~nm}$ is mainly due to the choice of the normalization value, and reflects the accuracy of that determination. For thicker films the first deviation progressively shifts to higher $t$.

\section{DISCUSSION}

The first point to be discussed is whether the measured values of $J_{c 0}$ agree with the theoretical estimates for $J_{d p}$.

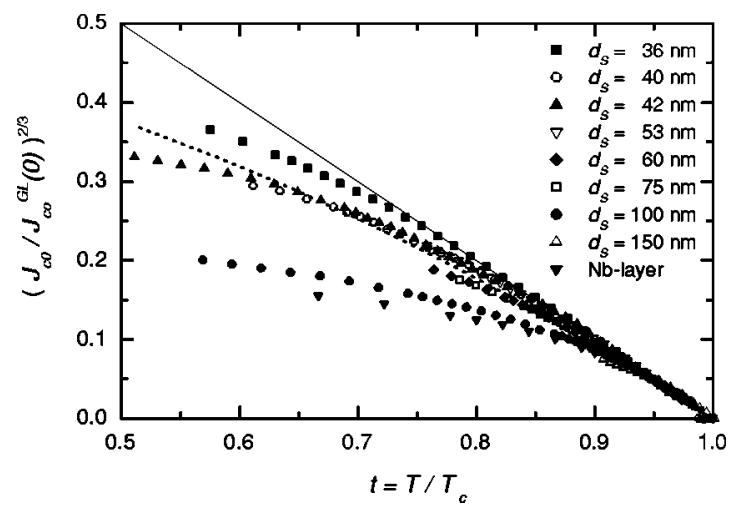

FIG. 4. $\left[J_{c 0} / J_{c 0}^{G L}(0)\right]^{2 / 3}$ vs $t=T / T_{c}$ for $\mathrm{Fe} / \mathrm{Nb} / \mathrm{Fe}$ trilayers with different thickness $d_{s}$ of the $\mathrm{Nb}$ layer, as indicated. The drawn line indicates the GL behavior, the dotted line is the result of the full calculation. 


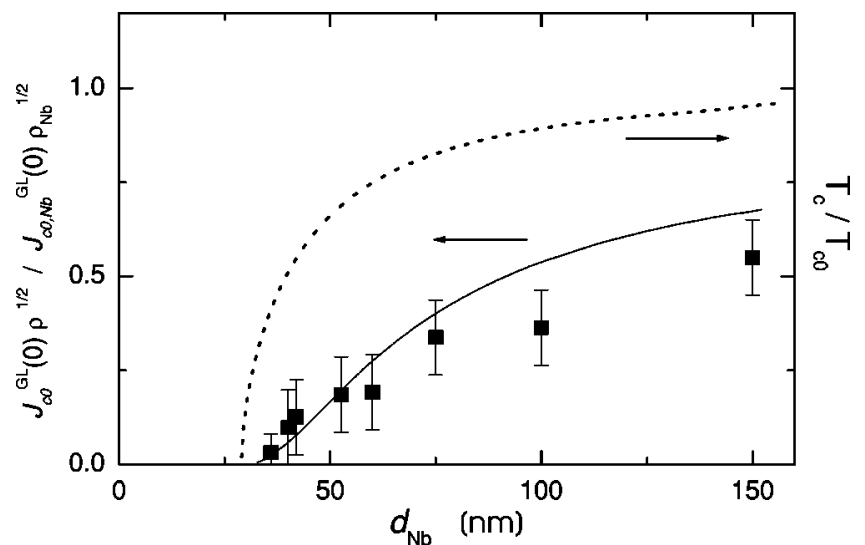

FIG. 5. $J_{c 0}^{G L}(0) \rho^{1 / 2}$ of the $\mathrm{Fe} / \mathrm{Nb} / \mathrm{Fe}$ trilayers scaled on the value of the single $\mathrm{Nb}$ layer vs superconducting layer thickness $d_{\mathrm{Nb}}$. The result of the model calculations for $\gamma=34.6, \gamma_{b}=42$ is also plotted (solid line) as well as the dependence of the critical temperature $T_{c} / T_{c 0}$ on $d_{\mathrm{Nb}}$ (dashed line) for the same parameters.

The absolute value of $J_{d p}^{G L}(0)$ can be calculated with Eq. 5 . The materials constants for $\mathrm{Nb}$ are well documented; ${ }^{13}$ we use the values $v_{F}=5.6 \times 10^{5} \mathrm{~m} / \mathrm{s}$ and $\rho l=3.75$ $\times 10^{-16} \Omega \mathrm{m}^{2}$. Equation (5) then yields for the $\mathrm{Nb}$ film $J_{d p, \mathrm{Nb}}^{G L}(0)=1.70 \times 10^{12} \mathrm{~A} / \mathrm{m}^{2}$, which is quite close to the experimentally determined value of $J_{c 0, \mathrm{Nb}}^{G L}(0)=1.5$ $\times 10^{12} \mathrm{~A} / \mathrm{m}^{2}$ (see Table I). It is also in good correspondence with the data presented by Ando et al. ${ }^{14}$ on films with a thickness of $100 \mathrm{~nm}$ and different strip widths between $0.1 \mu \mathrm{m}$ and $10 \mu \mathrm{m}$, who found a fitted value $J_{d p, \mathrm{Nb}}^{G L}(0)$ $=1.26 \times 10^{12} \mathrm{~A} / \mathrm{m}^{2}$. It appears that the depairing current is directly probed by the measurement of $J_{c 0}$.

Next we consider the dependence of $J_{c 0}^{G L}(0)$ on the superconducting Nb-layer thickness $d_{\mathrm{Nb}}$. As Eq. (5) shows, $J_{d p}(0)$ is proportional to $\sqrt{1 / \rho_{\mathrm{Nb}}}$. Since $\rho_{\mathrm{Nb}}$ of the samples differs, this leads to some variation in the expected value for $J_{d p}(0)$ that can be taken into account by multiplying $J_{c 0}^{G L}(0)$ by $\rho_{\mathrm{Nb}}^{1 / 2}$. Normalizing this value to the single $\mathrm{Nb}$ film yields the dependence on $d_{\mathrm{Nb}}$ as shown in Fig. 5. $J_{c 0}^{G L}(0)$ in the trilayers is clearly reduced with respect to the bulk $\mathrm{Nb}$ value and increases with increasing $d_{\mathrm{Nb}}$, but much more slowly than $T_{c}$ does. The correspondence with theory is good at low $d_{\mathrm{Nb}}$, with some deviations above $d_{\mathrm{Nb}} \approx 75 \mathrm{~nm}$. This coincides with the findings on the temperature dependence of $J_{c 0}(t)$, shown in Fig. 4: for small $d_{\mathrm{Nb}}$ there are only small deviations in the whole measured temperature regime, for large $d_{\mathrm{Nb}}$ the deviations are large below $t=0.9$. This suggests that at high $d_{\mathrm{Nb}}$ the extrapolation for $J_{c 0}^{G L}(0)$ leads to somewhat underestimated values. In essence, we conclude that the model used to describe the depression of $T_{c}$ in $F / S / S$ trilayers also adequately describes the behavior of $J_{d p}$.

A second point to be addressed is the spatial distribution of the transport current. In order to determine the depairing current it is usually understood that two conditions have to be fulfilled: ${ }^{7,15}$ the current has to be distributed uniformly over the strip, and the width $w$ should be small enough to preclude vortex formation and motion. In terms of penetration depth $\lambda(t)$, strip thickness $d_{s}$, and Ginzburg-Landau coherence length $\xi(t)$ this means

$$
\begin{gathered}
w<\lambda_{e f f}(t)= \begin{cases}\lambda(t), & d_{s}<\lambda_{e f f}(t) \\
\lambda^{2}(t) / d_{s}, & d_{s}>\lambda_{e f f}(t),\end{cases} \\
w<4.4 \xi(t)=4.4 \xi(0) /(1-t)^{1 / 2} .
\end{gathered}
$$

Estimating $\lambda(0)$ from $\lambda(0)=1.05 \times 10^{-3} \sqrt{\rho_{0} / T_{c}}$ we find it ranges between 67 and $113 \mathrm{~nm}$. Both conditions mean for all samples $1-t<10^{-4}$, much smaller than the region where $J_{c 0}(t) \propto(1-t)^{3 / 2}$ (Fig. 4), and the question is valid whether the current is uniform, as has implicitly been assumed in the analysis.

Qualitatively, current is expected to pile up at the edges of the strip in order to minimize the self field inside. The edge current will then sooner reach the value of $I_{d p}$. By using $J_{d p}=I_{d p} /(w d)$, this would lead to underestimating the real value of $J_{d p}$. From the close agreement between the experimental and theoretical values this does not appear to be the case. Quantitatively, the situation can be assessed that the self field of the sample is completely screened $\left(B_{z}=0\right.$ in the sample). The current distribution is then given by ${ }^{16}$

$$
J(x)=\frac{I_{T}}{\pi d \sqrt{W^{2}-x^{2}}}, \quad-W<x<W,
$$

where $I_{T}$ is the transport current through the sample, $x$ is in the direction of the width $w$ of the film, $x=0$ is in the middle of the film and $2 W=w$. According to this formula, the current diverges at the edges of the film. It can be assumed, however, that the field penetrates over a distance $d / 2$ from the edges, but is kept out of the rest of the sample by the screening current. Then, the current within $d / 2$ from the edges can be set equal to $J_{d p}^{G L}(0)$ and beyond $d / 2$ it decreases according to Eq. (9). The following calculation can be done for the $\mathrm{Nb}$ film. The transport current $I_{T}$ in the screened part of the strip can be calculated from Eq. 9 by using

$$
J(x=W-d / 2)=J_{d p}^{G L}(0) .
$$

The total current $I$ including the edges is given by

$$
\begin{aligned}
I / d & =2(d / 2) J_{d p}^{G L}(0)+\int_{-W+d / 2}^{W-d / 2} \frac{I_{T}}{\pi d \sqrt{W^{2}-x^{2}}} \\
& =d J_{d p}^{G L}(0)\left(1+\sqrt{\frac{w}{2 d}}\left[\sin ^{-1} \frac{x}{|W|}\right]_{-W+d / 2}^{W-d / 2}\right) .
\end{aligned}
$$

The ratio $[I /(w d)] / J_{d p}^{G L}(0)$, which can be calculated from Eq. 11, gives the fraction of $J_{d p}^{G L}(0)$ that would be actually measured as the critical current under the given current distribution, where the depairing current is reached at the edges. It can be easily seen that it equals 1 when the current is uniform. For the $\mathrm{Nb}$ film with $w$ and $d$ as given in Table I, Equation (11) yields a fraction of 0.11, an order of magnitude below what is actually measured. The conclusion is that $J(x)$ is much more uniformly distributed than might be expected. The reason is probably that a magnetic field and moving vortices exist in the film, indicated by a voltage onset below 
the jump to the normal state. This breaks up the Meissner state, causes a much more uniform current distribution, and allows the correct determination of the depairing current over a much larger region than expected on the basis of the condition $w<\lambda, \xi$. Still, the deviations of $J_{c 0}(t)$ compared to the theoretical behavior at higher $d_{\mathrm{Nb}}$ in the $\mathrm{Fe} / \mathrm{Nb} / \mathrm{Fe}$ trilayers may be due to the low value of $\lambda$ and inhomogeneities in the current distribution at these high thicknesses. At low thicknesses, there are two effects that increase $\lambda$ above the bulk value. First, for $d_{s}<\lambda$ (around $d_{\mathrm{Nb}} \approx 75 \mathrm{~nm}$ ) the effective penetration depth increases according to $\lambda_{\text {eff }}=\lambda(0)^{2} / d$, and can become significantly higher than $\lambda(0)$. Second, the suppression of the order parameter as measured by the decrease of $T_{c} / T_{c 0}$ results in a higher value for $\lambda(0)$. From that point of view the full agreement between the measured and calculated values of $J_{c 0}(t)$ at the lowest thicknesses is not surprising. A final remark concerns the apparent absence of effects from the magnetic dipole field of the $F$ layers on the $S$ layer. In principle, the magnetization of the $F$ layers is in plane because of the small thickness, which means that the magnetic fields penetrating into the $S$ layer will be small. This would even be the case for the magnetization perpendicular to the film, because of the large demagnetization factor. These are the reasons why both parallel and perpendicular critical fields of $F / S / F$ systems can be well described by proximity-effect theory only. ${ }^{17,18}$ Still, magnetic domain structure in the sample could lead to appreciable stray fields at the domain walls if the domains are large enough. We suppose this would give rise to Abrikosov vortices, which can move and help to homogenize the current. It would mean that the onset of voltage/dissipation might depend on the domain structure of the magnetic layer. This point is currently under investigation.

\section{SUMMARY}

In this paper we have addressed the question of the value of the superconducting depairing current in $F / S / F$ trilayers with varying $d_{s}$, where the superconducting order parameter is inhomogeneously suppressed by the pair breaking in the $F$ layers. The same model that adequately describes the suppression of $T_{c}$ with decreasing $d_{s}$ with two parameters (proximity strength $\gamma$ and interface transparency $\gamma_{b}$ or $T$ ) can also be used to compute the suppression of the depairing current. Measurements of the zero-field critical current $J_{c 0}$ (as defined by the current where the resistance jumps to the normal-state value) in thin strips of $\mathrm{Fe} / \mathrm{Nb} / \mathrm{Fe}$ show that the temperature dependence near $T_{c}$ is as expected for the depairing current. Also the absolute value of $J_{c 0}$ of single $\mathrm{Nb}$ films is close to the theoretically expected value and the measured suppression of $J_{c 0}$ in the trilayers follows the calculated behavior. We conclude that the current distribution is homogeneous and that the depairing current is measured, even though the strip widths are larger than the superconducting penetration depth and coherence length. Also, the proximity-effect model correctly describes the shape of the order parameter, at least in the superconducting layer. These findings can be of use in experiments on the effect of injecting polarized quasiparticles.

\section{ACKNOWLEDGMENTS}

This work was part of the research program of the "Stichting voor Fundamenteel Onderzoek der Materie (FOM)," which is financially supported by NWO. A.A.G. acknowledges support by NWO in the framework of the RussianDutch collaboration program, Grant No. 047-005-01. We would like to thank P. H. Kes for helpful discussions and K. Temst (Leuven) for performing x-ray diffraction experiments.

\section{APPENDIX A: CALCULATION OF $\boldsymbol{J}_{d p}$}

We assume that the dirty limit conditions are fulfilled in both $S$ and $F$ layers, so that the $F / S$ bilayer can be described by the Usadel equations. In the absence of a depairing current in the $S$ layer, and in the regime of large exchange energy in the ferromagnet $\left(E_{e x} \gg k_{B} T_{c}\right)$ these equations were discussed extensively by Buzdin et al. ${ }^{19}$ (see also Demler et $a l^{20}{ }^{20}$. Here we rewrite these equation in $\theta$ parametrization ( $F=\sin \theta, G=\cos \theta$ ) and include the pair-breaking effects by current along the $S$ film:

$$
\begin{gathered}
\xi_{S}^{2} \frac{d^{2}}{d z^{2}} \theta_{S}(z)-\tilde{\omega} \sin \theta_{S}(z)+\Delta(z) \cos \theta_{S}(z)=0, \\
\xi_{F}^{2} \frac{d^{2}}{d z^{2}} \theta_{F}(z)-i \sin \theta_{F}(z)=0, \\
\Delta \ln \left(T / T_{c}\right)+\pi T \sum_{\omega_{n}}\left(\frac{\Delta}{\left|\omega_{n}\right|}-\sin \theta_{S}\right)=0,
\end{gathered}
$$

where $\omega_{n}=\pi(2 n+1) T / T_{c}$ is the normalized Matsubara frequency, $\tilde{\omega}=\left|\omega_{n}\right|+Q^{2} \cos \theta(z), \Delta$ is the pair potential in a superconductor normalized to $\pi T_{c}, \xi_{S}=\left(\hbar D_{S} / 2 \pi T_{c}\right)^{1 / 2}$, $\xi_{F}=\left(\hbar D_{F} / 2 E_{e x}\right)^{1 / 2}$, and $D_{F, S}$ are the coherence lengths and the electronic diffusion coefficients in $F$ and $S$ metals. Moreover, $Q=\xi_{s} \partial \chi / \partial x$ is the normalized gradient-invariant superfluid velocity in the $x$ direction, with $\chi$ the phase of the pair potential $\Delta$. There are two sources of pair breaking in the problem, the volume one by the current and the surface one by the ferromagnet. The latter is described by the boundary conditions at the $F S$ interface $(z=0)$,

$$
\begin{gathered}
\xi_{S} \frac{d}{d z} \theta_{S}=\gamma \xi_{F} \frac{d}{d z} \theta_{F}, \\
\gamma_{b} \xi_{F} \frac{d}{d z} \theta_{F}=\sin \left(\theta_{S}-\theta_{F}\right),
\end{gathered}
$$

where the parameter $\gamma=\rho_{S} \xi_{S} / \rho_{F} \xi_{F}$ describes the strength of the suppression of superconductivity in $S$ by the ferromagnet.

The parameter $\gamma_{b}$ describes the effect of boundary transparency (coupling strength) between the layers. In the $N S$ case, when the decoupling is due to the presence of an additional potential barrier at the interface, $\gamma_{b}=R_{B} / \rho_{F} \xi_{F}$, with $R_{B}$ the normal-state resistance of the $N / S$ interface. ${ }^{21}$ In the $F / S$ bilayer there is no general microscopic derivation for $\gamma_{b}$, combining the effect of exchange splitting and an addi- 
tional interface barrier. A simple estimate is still possible, when the exchange splitting is the main cause for intransparency. ${ }^{6} \quad$ Then $\gamma_{b}=(2 / 3)\left(l_{F} / \xi_{F}\right)\left\langle\left(1-T_{A}\right) / T_{A}\right\rangle$, where $T_{A}$ is the transmission probability of scattering between the majority and minority spin subbands, i.e., the probability of Andreev reflection. This process is implicitly described by the boundary condition $\gamma_{b} \xi_{F}(d / d z) \theta_{F}=\sin \left(\theta_{S}\right.$ $-\theta_{F}$ ) since $\theta_{F}$ is off diagonal in spin indices. Here the brackets $\langle\cdots\rangle$ denote the Fermi-surface averaging, which is generally proportional to the overlap area of the projections of different spin subbands onto the contact plane. ${ }^{22,23}$ As a result, $T_{A}$ drops roughly linearly (for spherical Fermi surfaces) as a function of $E_{e x}$, both for ballistic and diffusive interfaces. ${ }^{24}$ The supercurrent density is given by

$$
J_{s}(z, Q)=\frac{2 \pi \sigma_{s}}{e} Q T \sum_{\omega_{n}} \sin ^{2} \theta_{s} .
$$

Since the superconducting pair potential $\Delta$ and the Green's function $\theta_{s}$ are suppressed by the superflow $Q$, the depen- dence $J_{s}(Q)$ must be found self-consistently. In the wellknown spatially homogeneous case ${ }^{25}$ the function $J_{s}(Q)$ behaves nonmonotonously: the supercurrent $J_{s}$ increases with $Q$ at small $Q$, then reaches a maximum and finally drops to zero, when $\Delta$ is fully suppressed by current. The depairing current is defined as the maximum of $J_{s}(Q)$. A similar situation holds in the spatially inhomogeneous case considered here, with the difference that the solutions for $\theta(z)$ and $\Delta(z)$ of the proximity-effect problem [Eqs. (A1)-(A3)] should be calculated self-consistently for a given $Q$ using the boundary conditions at the $F S$ interface [Eqs. (A4) and (A5)]. This problem is solved numerically by the method applied previously to NS bilayers and are described in detail in Ref. 26. Then the local $z$-dependent supercurrent density $J_{s}(z, Q)$ is calculated from Eq. (A6) by summing the solutions $\sin ^{2} \theta_{s}$ over $\omega_{n}$. Finally the density is averaged over film thickness $J_{s}(Q)=d_{s}^{-1} \int_{0}^{d_{s}} J_{s}(z, Q) d z$ and the depairing current is found from the maximum of the dependence of $\left\langle J_{s}\right\rangle$ on $Q$.
*Corresponding author. Email address: aarts@phys.leidenuniv.nl

${ }^{1}$ R. Meservey and P. M. Tedrow, Phys. Rep. 238, 173 (1994).

${ }^{2}$ V. A. Vasko, V. A. Larkin, P. A. Kraus, K. R. Nikolaev, D. E. Grupp, C. A. Nordman, and A. M. Goldman, Phys. Rev. Lett. 78, 1134 (1997).

${ }^{3}$ Z. W. Dong, R. Ramesh, T. Venkatesan, M. Johnson, Z. Y. Chen, S. P. Pai, V. Talyansky, R. P. Sharma, R. Shreekala, C. J. Lobb, and R. L. Greene, Appl. Phys. Lett. 71, 1718 (1997).

${ }^{4}$ N.-C. Yeh, R. P. Vasquez, C. C. Fu, A. V. Samoilov, Y. Li, and K. Vakili, Phys. Rev. B 60, 10522 (1999).

${ }^{5}$ J. M. de Teresa, A. Barthélémy, A. Fert, J. P. Contour, R. Lyonnet, F. Montaigne, P. Seneor, and A. Vaurès, Phys. Rev. Lett. 82, 4288 (1999).

${ }^{6}$ J. Aarts, J. M. E. Geers, E. Brück, A. A. Golubov, and R. Coehoorn, Phys. Rev. B 56, 2779 (1997).

${ }^{7}$ J. Romijn, T. M. Klapwijk, M. J. Renne, and J. E. Mooij, Phys. Rev. B 26, 3648 (1982).

${ }^{8}$ M. Yu. Kupriyanov and V. F. Lukichev, Fiz. Nizk. Temp. 6, 445 (1980) [Sov. J. Low Temp. Phys. 6, 210 (1980)].

${ }^{9}$ Th. Mühge, K. Theis-Bröhl, K. Westerholt, H. Zabel, N. N. Garif'yanov, Yu. V. Goryunov, I. A. Garifullin, and G. G. Khaliullin, Phys. Rev. B 57, 5071 (1998).

${ }^{10}$ G. Verbanck, C. D. Potter, V. Metlushko, R. Schad, V. V. Moshchalkov, and Y. Bruynseraede, Phys. Rev. B 57, 6029 (1998).

${ }^{11}$ A. I. Buzdin, A. V. Vedyayev, and N. V. Ryzhanova, Europhys. Lett. 48, 686 (1999).
${ }^{12}$ L. Tagirov, Phys. Rev. Lett. 83, 2058 (1999).

${ }^{13}$ H. W. Weber, E. Seidl, C. Laa, E. Schachinger, M. Prohammer, A. Junod, and D. Eckert, Phys. Rev. B 44, 7585 (1991).

${ }^{14}$ Y. Ando, H. Kubota, S. Tanaka, M. Aoyagi, H. Akoh, and S. Takada, Phys. Rev. B 47, 5481 (1993).

${ }^{15}$ K. K. Likharev, Rev. Mod. Phys. 51, 101 (1997).

${ }^{16}$ E. Zeldov, J. R. Clem, M. McElfresh, and M. Darwin, Phys. Rev. B 49, 9802 (1994).

${ }^{17}$ P. Koorevaar, Y. Suzuki, R. Coehoorn, and J. Aarts, Phys. Rev. B 49, 441 (1994).

${ }^{18}$ P. Koorevaar, R. Coehoorn, and J. Aarts, Physica C 248, 61 (1995).

${ }^{19}$ A. I. Buzdin, B. Vujicic, and M. Yu. Kupriyanov, Zh. Éksp. Teor. Fiz. 101, 231 (1992) [Sov. Phys. JETP 74, 124 (1992)].

${ }^{20}$ E. A. Demler, G. B. Arnold, and M. R. Beasley, Phys. Rev. B 55, 15174 (1997).

${ }^{21}$ M. Yu. Kuprianov and V. F. Lukichev, Zh. Éksp. Teor. Fiz. 94, 139 (1988) [Sov. Phys. JETP 67, 1163 (1988)].

${ }^{22}$ M. J. M. de Jong and C. W. J. Beenakker, Phys. Rev. Lett. 74, 1657 (1995).

${ }^{23}$ I. I. Mazin, Phys. Rev. Lett. 83, 1427 (1999).

${ }^{24}$ I. I. Mazin, A. A. Golubov, and B. Nadgorny, cond-mat/0010326 (unpublished).

${ }^{25}$ J. Bardeen, Rev. Mod. Phys. 34, 667 (1962).

${ }^{26}$ A. A. Golubov, E. P. Houwman, J. G. Gijsbertsen, V. M. Krasnov, J. Flokstra, H. Rogalla, and M. Yu. Kupriyanov, Phys. Rev. B 51, 1073 (1995). 